\title{
When COVID-19 will decline in India? Prediction by combination of recovery and case load rate
}

\author{
Atanu Bhattacharjee ${ }^{\mathrm{a}, \mathrm{b}}$, Mukesh Kumar ${ }^{\mathrm{c}, *}$, Kamalesh Kumar Patel $^{\mathrm{d}}$ \\ ${ }^{a}$ Section of Biostatistics, Centre for Cancer Epidemiology, TMC, Mumbai, India \\ ${ }^{\mathrm{b}}$ Homi Bhaba National Institute, Mumbai, India \\ ${ }^{c}$ Department of Statistics, MMV, Banaras Hindu University, Varanasi, India \\ ${ }^{\mathrm{d}}$ Department of Public Health, Indian Institute of Health Management Research, Jaipur, India
}

A R T I C L E I N F O

\section{Keywords:}

COVID-19

Recovery rate

Case load rate

Death rate

India

\begin{abstract}
A B S T R A C T
Background: The World Health Organization (WHO) declared COVID-19 as a pandemic on March 11, 2020. There is sudden need of statistical modeling due to onset of COVID-19 pandemic across the world. But health planning and policy requirements need the estimates of disease problem from clinical data.

Objective: The present study aimed to predict the declination of COVID-19 using recovery rate and case load rate on basis of available data from India.

Methods: The reported COVID-19 cases in the country were obtained from website (https://datahub.io/core/ covid-19\#resource-covid-19_zip/). The confirmed cases, recovered cases and deaths were used for estimating recovery rate, case load rate and death rate till June 04, 2020.

Results: A total of 216919 confirmed cases were reported nationwide in India on June 04, 2020. It is found that the recovery rate increased to $47.99 \%$ and case load rate decreased to $49.21 \%$. Death rate is found to be very low $2.80 \%$. Accordingly, coincidence of the difference of case load rate and recovery rate (delta) will reveal a declination in expected COVID-19 cases.

Conclusion: The epidemic in the country was mainly caused by the movement of people from various foreign countries to India. Lockdown as restricting the migration of population and decision taken by the government to quarantine the population may greatly reduce the risk of continued spread of the epidemic in India. This study predicts that when the case load rate gets lesser than recovery rate, there after COVID-19 patients would be started to decline.
\end{abstract}

\section{Introduction}

Since, December 2019, a pneumonia infection broke out in Wuhan, Hubei province and spread in entire China and many other countries. Chinese health authorities observed notoriously a cluster of pneumonia cases of unknown aetiology. ${ }^{1}$ Relations between the key cases and the city's South China Sea food market were found. The chance of another zoonosis or severe acute respiratory syndrome (SARS) outbreak was at the top of the priority, investigations were attempted which result in recognition of a novel coronavirus, SARS-CoV-2 (formerly 2019-nCoV), as the agent has entered as international outbreak in Hubei. China has revealed total of 72,528 confirmed cases till February 17, 2020..$^{2,3}$ The novel coronavirus 2019 (COVID-19) has spread across 210 nations and regions with 1.2 million confirmed cases and 67594 deaths reported on April 6, 2020. The WHO declared public health as an emergency at international level. The worldwide community gets worried about
COVID-19 and its impact on public health. WHO is trying to control the impact of pandemic through identification, testing, treatment of patients, clinical trials for drugs and development of new vaccines. ${ }^{4}$ In India, first case of COVID -19 was reported on January 30, 2020 who returned from Wuhan, China. Considering the first case as a matter of great worry, screening of traveller at airport had been started, immediately Chinese visas had been cancelled, and people who were found affected with COVID-19 had been quarantined. ${ }^{5}$ The Ministry of Health and Family Welfare (MoHFW) of India had primarily warned to avoid travelling to China and advised quarantine of those returning from China. ${ }^{6}$ However, India is at high risk forbeing the second highest populated country in the world. Most of the studies show the prediction of COVID -19 through modeling for growth of infected population. In the absence of a licensed vaccine or effective therapeutics for COVID19 , other advises from hand cleanliness to quarantine, a basic strategy to control spread of epidemic and alleviation mediation towards the

\footnotetext{
* Corresponding author. Department of Statistics, MMV, Banaras Hindu University, Varanasi 221005, India.

E-mail address: mukesh.mmv@bhu.ac.in (M. Kumar).
} 
early detection and quarantine of cases can break the chain of transmission. The SARS-CoV-2 pandemic is currently a great challenge for researchers, clinicians, health-care workers, and decision makers. We depict the most striking difficulties for statisticians who need to provide support in this pandemic with their proficiency. Having a knowledge of the dynamics of case load rate and recovery rate of COVID-19 can enhance the basic understanding to a large extent based on the current patterns of the severity of the epidemic. As COVID-19 cases are increasing day-by-day, case load rate will be of utmost importance in predicting the declination of the epidemic. In this article, we want to predict the date when the recovery rate of patients would be more than case load rate in India. This date is defined as disease declined date.

\section{Materials and methods}

\subsection{Data sources}

Data safety, patient's consent, ethical approvals are essential in nonpandemic circumstance but there are administrative barriers to get access to clinical data. Pandemic circumstances need precise handling of these issues and should be examined nationwide. Clinical data are extremely time-dependent and involve progressive statistical methods. ${ }^{7}$ Data has been obtained with parameters as confirmed cases, recovered cases and deaths from the online website (https://datahub.io/core/ covid-19\#resource-covid-19_zip/). Total 216919 confirmed cases, 104107 recovered cases and 6075 deaths were- reported till June 04, 2020 and included in the study.

\section{Methodology}

The whole analysis has been carried out with data available on cumulative confirmed cases, recovered cases and number of deaths. Recovery rate is defined as the proportion of number of recovered cases to confirmed cases, while the case load rate is defined as the proportion of case load to confirmed cases. Following formulae have been used for estimation purpose in (Table 1).

Recovery rate $=\frac{\begin{array}{l}\text { Recovered } \\ \text { cases } \\ \text { Confirmed } \\ \text { cases }\end{array}}{200}$

$$
\begin{aligned}
\text { Case load }= & \text { Confirmed } \\
& \text { cases }- \text { Recovered } \\
& \text { cases }- \text { Deaths }
\end{aligned}
$$

$$
\begin{aligned}
\text { Case load rate }= & \frac{\text { Case load }}{\text { Confirmed }} * 100 \\
& \text { cases }
\end{aligned}
$$

$$
\text { Death rate }=\frac{\text { Deaths }}{\begin{array}{l}
\text { Confirmed } \\
\text { cases }
\end{array}} * 100
$$

Delta $=$

$$
\text { Case load rate - Recovery rate }
$$

\section{Result}

Total 2169191 confirmed cases and 104107 recovered cases were involved in the study. Recovery rate had been estimated as $47.99 \%$ on the basis of confirmed and recovered cases. Total case load was found to be 106737 cases and case load rate was estimated as $49.21 \%$. Death rate was obtained as $2.80 \%$ based on 6075 dead patients. Delta, being the difference of case load rate and recovery rate was obtained as $1.21 \%$ on June 04,2020 as evident from (Table 1). The value of delta has been estimated from January 30, 2020 to June 04, 2020. It has been predicted that the value of delta would be least on June 12, 2020; it means the difference between case load rate and recovery rate would approach a negligible value. This indicates that confirmed cases expected would start to decline thereafter with respect to recovered cases as shown in (Fig. 1).

\section{Discussion}

2019-nCoV is a new coronavirus, which is unique in relation to SARS infections and has never been found in the human population afore. ${ }^{8}$ The cases caused by $2019-\mathrm{nCoV}$ has evidently confirmed strong relational correspondence ability and that the population is usually vulnerable. ${ }^{9}$ This study deals with the estimation of the recovery rate and case load rate in India. The impact might be decreased with the prevention and control actions implemented by the Indian government. The results of this study suggest that actively adopting a containment strategy will continue to increase the control of the epidemic in India . Lockdown quarantine, clinical perception and suspected cases and imposing restrictions on population movement (complete lockdown) as well will help to reduce the risk of COVID -19 in India. ${ }^{10}$ Explicitly various kinds of models have been utilized by the investigators keeping in view the main objective for which the models have been created with conclusion. The confirmed cases and evolving reports of better understanding and better expectation of the imminent estimates from other nations have resulted in emerging mathematical tools for forcasting the validity of different preventive and management policies. In India, initially the recovery rate has been decreasing till April 10, 2020 with some peaks in between. After that, it is approximately increasing with its maximum value of $47.99 \%$ on June 04,2020 . This finding suggests that lockdown strategy has played a great role in reducing the spread of the disease. Similarly, case load rate is inconsistent in starting till April 10, 2020. After that, it is continuously decreasing and is least on June 04, 2020 having a value of $49.21 \%$. In this article, proposed models have resulted in findings regarding the recovery rate and case load rate associated with COVID-19 disease using application of Statistics. Estimates of delta have been used for prediction purpose.

\section{Conclusion}

Investigations have been made on the issue of COVID-19 pandemic spread in India in the current challenging scenario. Trend knowledge has been observed with the help of recovery rate and case load rate obtained for the data available. The various strategies implemented as lockdown, quarantine of population have played a significant role in reducing the risk of spread of epidemic. This study predicts that when the case load rate gets lesser than recovery rate, there after COVID-19 patients would start to decline.

\section{Limitation}

This study is restricted within the time period of analysis till June 04, 2020 in India. Different models were considered to estimate COVID19 extent, and claimed to be precise, however they critically revealed data gaps and prerequisite to adjust difficult variables such as effect and uncertainty of lockdown, risk factors and social distancing might be reflected before generalizing the findings.

\section{Funding}

The authors did not receive any funding for this work. 
Table 1

Estimate of recovery rate, case load rate based on confirmed and recovered cases.

\begin{tabular}{|c|c|c|c|c|c|c|c|c|}
\hline Date & Confirmed cases & Recovered cases & Deaths & Recovery rate & Case load & Case load rate & Death rate & Delta \\
\hline 04-03-2020 & 28 & 3 & 0 & 10.71 & 25 & 89.29 & 0.00 & 78.57 \\
\hline 05-03-2020 & 30 & 3 & 0 & 10.00 & 27 & 90.00 & 0.00 & 80.00 \\
\hline 06-03-2020 & 31 & 3 & 0 & 9.68 & 28 & 90.32 & 0.00 & 80.65 \\
\hline 07-03-2020 & 34 & 3 & 0 & 8.82 & 31 & 91.18 & 0.00 & 82.35 \\
\hline 08-03-2020 & 39 & 3 & 0 & 7.69 & 36 & 92.31 & 0.00 & 84.62 \\
\hline 09-03-2020 & 46 & 3 & 0 & 6.52 & 43 & 93.48 & 0.00 & 86.96 \\
\hline 10-03-2020 & 58 & 3 & 0 & 5.17 & 55 & 94.83 & 0.00 & 89.66 \\
\hline $11-03-2020$ & 60 & 3 & 0 & 5.00 & 57 & 95.00 & 0.00 & 90.00 \\
\hline $12-03-2020$ & 74 & 3 & 0 & 4.05 & 71 & 95.95 & 0.00 & 91.89 \\
\hline 13-03-2020 & 81 & 3 & 1 & 3.70 & 77 & 95.06 & 1.23 & 91.36 \\
\hline $14-03-2020$ & 84 & 10 & 2 & 11.90 & 72 & 85.71 & 2.38 & 73.81 \\
\hline $15-03-2020$ & 110 & 13 & 2 & 11.82 & 95 & 86.36 & 1.82 & 74.55 \\
\hline $16-03-2020$ & 114 & 13 & 2 & 11.40 & 99 & 86.84 & 1.75 & 75.44 \\
\hline $17-03-2020$ & 137 & 14 & 3 & 10.22 & 120 & 87.59 & 2.19 & 77.37 \\
\hline $18-03-2020$ & 151 & 14 & 3 & 9.27 & 134 & 88.74 & 1.99 & 79.47 \\
\hline $19-03-2020$ & 173 & 20 & 4 & 11.56 & 149 & 86.13 & 2.31 & 74.57 \\
\hline 20-03-2020 & 223 & 23 & 4 & 10.31 & 196 & 87.89 & 1.79 & 77.58 \\
\hline 21-03-2020 & 283 & 23 & 4 & 8.13 & 256 & 90.46 & 1.41 & 82.33 \\
\hline $22-03-2020$ & 360 & 24 & 7 & 6.67 & 329 & 91.39 & 1.94 & 84.72 \\
\hline 23-03-2020 & 433 & 24 & 7 & 5.54 & 402 & 92.84 & 1.62 & 87.30 \\
\hline 24-03-2020 & 519 & 40 & 9 & 7.71 & 470 & 90.56 & 1.73 & 82.85 \\
\hline $25-03-2020$ & 606 & 43 & 10 & 7.10 & 553 & 91.25 & 1.65 & 84.16 \\
\hline 26-03-2020 & 694 & 45 & 15 & 6.48 & 634 & 91.35 & 2.16 & 84.87 \\
\hline 27-03-2020 & 724 & 67 & 17 & 9.25 & 640 & 88.40 & 2.35 & 79.14 \\
\hline $28-03-2020$ & 909 & 80 & 19 & 8.80 & 810 & 89.11 & 2.09 & 80.31 \\
\hline 29-03-2020 & 1024 & 96 & 27 & 9.38 & 901 & 87.99 & 2.64 & 78.61 \\
\hline $30-03-2020$ & 1251 & 102 & 31 & 8.15 & 1118 & 89.37 & 2.48 & 81.22 \\
\hline 31-03-2020 & 1397 & 124 & 35 & 8.88 & 1238 & 88.62 & 2.51 & 79.74 \\
\hline 01-04-2020 & 1834 & 144 & 41 & 7.85 & 1649 & 89.91 & 2.24 & 82.06 \\
\hline 02-04-2020 & 2069 & 156 & 53 & 7.54 & 1860 & 89.90 & 2.56 & 82.36 \\
\hline 03-04-2020 & 2547 & 163 & 62 & 6.40 & 2322 & 91.17 & 2.43 & 84.77 \\
\hline 04-04-2020 & 3072 & 213 & 75 & 6.93 & 2784 & 90.63 & 2.44 & 83.69 \\
\hline 05-04-2020 & 3577 & 275 & 83 & 7.69 & 3219 & 89.99 & 2.32 & 82.30 \\
\hline 06-04-2020 & 4281 & 319 & 111 & 7.45 & 3851 & 89.96 & 2.59 & 82.50 \\
\hline 07-04-2020 & 4789 & 353 & 124 & 7.37 & 4312 & 90.04 & 2.59 & 82.67 \\
\hline 08-04-2020 & 5274 & 411 & 149 & 7.79 & 4714 & 89.38 & 2.83 & 81.59 \\
\hline 09-04-2020 & 5865 & 478 & 169 & 8.15 & 5218 & 88.97 & 2.88 & 80.82 \\
\hline $10-04-2020$ & 6761 & 516 & 206 & 7.63 & 6039 & 89.32 & 3.05 & 81.69 \\
\hline $11-04-2020$ & 7529 & 653 & 242 & 8.67 & 6634 & 88.11 & 3.21 & 79.44 \\
\hline $12-04-2020$ & 8447 & 765 & 273 & 9.06 & 7409 & 87.71 & 3.23 & 78.66 \\
\hline $13-04-2020$ & 9352 & 980 & 324 & 10.48 & 8048 & 86.06 & 3.46 & 75.58 \\
\hline $14-04-2020$ & 10815 & 1190 & 353 & 11.00 & 9272 & 85.73 & 3.26 & 74.73 \\
\hline $15-04-2020$ & 11933 & 1344 & 392 & 11.26 & 10197 & 85.45 & 3.29 & 74.19 \\
\hline $16-04-2020$ & 12759 & 1515 & 420 & 11.87 & 10824 & 84.83 & 3.29 & 72.96 \\
\hline 17-04-2020 & 13835 & 1767 & 452 & 12.77 & 11616 & 83.96 & 3.27 & 71.19 \\
\hline $18-04-2020$ & 14792 & 2015 & 488 & 13.62 & 12289 & 83.08 & 3.30 & 69.46 \\
\hline $19-04-2020$ & 16116 & 2302 & 519 & 14.28 & 13295 & 82.50 & 3.22 & 68.21 \\
\hline $20-04-2020$ & 17656 & 2842 & 559 & 16.10 & 14255 & 80.74 & 3.17 & 64.64 \\
\hline 21-04-2020 & 18985 & 3260 & 603 & 17.17 & 15122 & 79.65 & 3.18 & 62.48 \\
\hline $22-04-2020$ & 20471 & 3960 & 652 & 19.34 & 15859 & 77.47 & 3.18 & 58.13 \\
\hline 23-04-2020 & 21700 & 4325 & 686 & 19.93 & 16689 & 76.91 & 3.16 & 56.98 \\
\hline $24-04-2020$ & 23452 & 4814 & 723 & 20.53 & 17915 & 76.39 & 3.08 & 55.86 \\
\hline 25-04-2020 & 24893 & 5210 & 779 & 20.93 & 18904 & 75.94 & 3.13 & 55.01 \\
\hline 26-04-2020 & 26605 & 5914 & 826 & 22.23 & 19865 & 74.67 & 3.10 & 52.44 \\
\hline 27-04-2020 & 28160 & 6362 & 886 & 22.59 & 20912 & 74.26 & 3.15 & 51.67 \\
\hline 28-04-2020 & 29834 & 7027 & 937 & 23.55 & 21870 & 73.31 & 3.14 & 49.75 \\
\hline 29-04-2020 & 31709 & 7797 & 1008 & 24.59 & 22904 & 72.23 & 3.18 & 47.64 \\
\hline 30-04-2020 & 33330 & 8373 & 1075 & 25.12 & 23882 & 71.65 & 3.23 & 46.53 \\
\hline 01-05-2020 & 34972 & 9065 & 1152 & 25.92 & 24755 & 70.79 & 3.29 & 44.86 \\
\hline 02-05-2020 & 37157 & 9951 & 1218 & 26.78 & 25988 & 69.94 & 3.28 & 43.16 \\
\hline 03-05-2020 & 40124 & 10887 & 1306 & 27.13 & 27931 & 69.61 & 3.25 & 42.48 \\
\hline 04-05-2020 & 42836 & 11762 & 1389 & 27.46 & 29685 & 69.30 & 3.24 & 41.84 \\
\hline 05-05-2020 & 46711 & 13161 & 1583 & 28.18 & 31967 & 68.44 & 3.39 & 40.26 \\
\hline $06-05-2020$ & 49391 & 14183 & 1694 & 28.72 & 33514 & 67.85 & 3.43 & 39.14 \\
\hline 07-05-2020 & 52952 & 15267 & 1783 & 28.83 & 35902 & 67.80 & 3.37 & 38.97 \\
\hline 08-05-2020 & 56342 & 16540 & 1886 & 29.36 & 37916 & 67.30 & 3.35 & 37.94 \\
\hline 09-05-2020 & 59662 & 17847 & 1981 & 29.91 & 39834 & 66.77 & 3.32 & 36.85 \\
\hline $10-05-2020$ & 62939 & 19358 & 2109 & 30.76 & 41472 & 65.89 & 3.35 & 35.14 \\
\hline $11-05-2020$ & 67152 & 20917 & 2206 & 31.15 & 44029 & 65.57 & 3.29 & 34.42 \\
\hline $12-05-2020$ & 70756 & 22455 & 2293 & 31.74 & 46008 & 65.02 & 3.24 & 33.29 \\
\hline $13-05-2020$ & 74281 & 24386 & 2415 & 32.83 & 47480 & 63.92 & 3.25 & 31.09 \\
\hline $14-05-2020$ & 78003 & 26235 & 2549 & 33.63 & 49219 & 63.10 & 3.27 & 29.47 \\
\hline $15-05-2020$ & 81970 & 27920 & 2649 & 34.06 & 51401 & 62.71 & 3.23 & 28.65 \\
\hline $16-05-2020$ & 85710 & 30153 & 2752 & 35.18 & 52805 & 61.61 & 3.21 & 26.43 \\
\hline
\end{tabular}

(continued on next page) 
Table 1 (continued)

\begin{tabular}{|c|c|c|c|c|c|c|c|c|}
\hline Date & Confirmed cases & Recovered cases & Deaths & Recovery rate & Case load & Case load rate & Death rate & Delta \\
\hline $17-05-2020$ & 90637 & 34109 & 2872 & 37.63 & 53656 & 59.20 & 3.17 & 21.57 \\
\hline $18-05-2020$ & 95759 & 36824 & 3029 & 38.45 & 55906 & 58.38 & 3.16 & 19.93 \\
\hline $19-05-2020$ & 100325 & 39174 & 3163 & 39.05 & 57988 & 57.80 & 3.15 & 18.75 \\
\hline $20-05-2020$ & 106750 & 42298 & 3303 & 39.62 & 61149 & 57.28 & 3.09 & 17.66 \\
\hline $21-05-2020$ & 112359 & 45300 & 3435 & 40.32 & 63624 & 56.63 & 3.06 & 16.31 \\
\hline $22-05-2020$ & 118447 & 48534 & 3583 & 40.98 & 66330 & 56.00 & 3.02 & 15.02 \\
\hline $23-05-2020$ & 125101 & 51784 & 3720 & 41.39 & 69597 & 55.63 & 2.97 & 14.24 \\
\hline $24-05-2020$ & 131868 & 54441 & 3867 & 41.28 & 73560 & 55.78 & 2.93 & 14.50 \\
\hline $25-05-2020$ & 138845 & 57721 & 4021 & 41.57 & 77103 & 55.53 & 2.90 & 13.96 \\
\hline $26-05-2020$ & 145380 & 60491 & 4167 & 41.61 & 80722 & 55.52 & 2.87 & 13.92 \\
\hline $27-05-2020$ & 151767 & 64426 & 4337 & 42.45 & 83004 & 54.69 & 2.86 & 12.24 \\
\hline $28-05-2020$ & 158333 & 67692 & 4531 & 42.75 & 86110 & 54.39 & 2.86 & 11.63 \\
\hline $29-05-2020$ & 165799 & 71106 & 4706 & 42.89 & 89987 & 54.27 & 2.84 & 11.39 \\
\hline $30-05-2020$ & 173763 & 82370 & 4971 & 47.40 & 86422 & 49.74 & 2.86 & 2.33 \\
\hline $31-05-2020$ & 182143 & 86984 & 5164 & 47.76 & 89995 & 49.41 & 2.84 & 1.65 \\
\hline 01-06-2020 & 190535 & 91819 & 5394 & 48.19 & 93322 & 48.98 & 2.83 & 0.79 \\
\hline 02-06-2020 & 198706 & 95527 & 5598 & 48.07 & 97581 & 49.11 & 2.82 & 1.03 \\
\hline 03-06-2020 & 207615 & 100303 & 5815 & 48.31 & 101497 & 48.89 & 2.80 & 0.58 \\
\hline 04-06-2020 & 216919 & 104107 & 6075 & 47.99 & 106737 & 49.21 & 2.80 & 1.21 \\
\hline
\end{tabular}

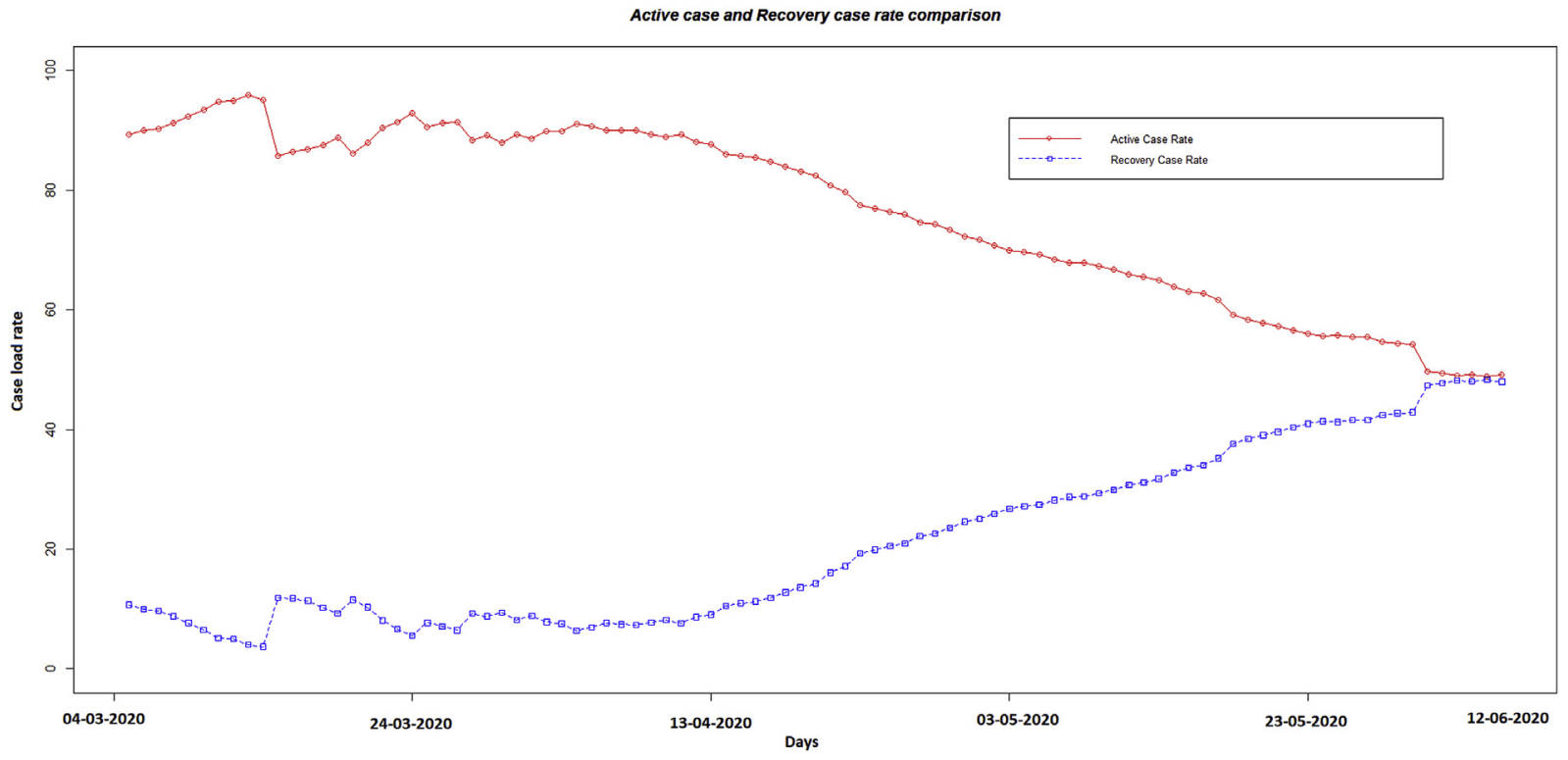

Fig. 1. Comparison of recovery rate and active case rate of COVID-19 patients in India.

\section{Declaration of competing interest}

The authors declare no conflict of interest.

\section{Acknowledgements}

The Authors would like to thanks to the editor in chief Professor Shally Awasthi and learned referee for their suggestions to improving the quality of contents of manuscript.

\section{References}

1. Liu T, Hu J, Xiao J, He G, Kang M, Rong Z, Zeng W. Time-varying transmission dynamics of Novel Coronavirus Pneumonia in China. BioRxiv. 2020.

2. Wu JT, Leung K, Leung GM. Now casting and forecasting the potential domestic and international spread of the 2019-nCoV outbreak originating in Wuhan, China: a modeling study. The Lancet. 2020;395(10225):689-697.
3. Chen W, Wang Q, Li YQ, Yu HL, Xia YY, Zhang ML, ... Yang XK. Early containment strategies and core measures for prevention and control of novel coronavirus pneumonia in China. Zhonghua yu fang yi xue za zhi [Chinese journal of preventive medicine]. 2020;54(3):1-6.

4. https://www.who.int/news-room/detail/30-01-2020-statement-on-the-secondmeeting-of-the-international-health-regulations-(2005)-emergency-committeeregarding-the-outbreak-of-novel-coronavirus-(2019-ncov).

5. https://boi.gov.in/content/advisory-travel-and-visarestrictions-related-covid-19-0.

6. https://www.mohfw.gov.in/.

7. Wolkewitz M, Puljak L. Methodological challenges of analysing COVID-19 data during the pandemic. 2020; 2020.

8. Lake MA. What we know so far: COVID-19 current clinical knowledge and research. Clinical Medicine, 2020:20(2):124.

9. Chen ZL, Zhang WJ, Lu Y, Guo C, Guo ZM, Liao CH, Lu JH. From SARS-CoV to 2019$\mathrm{nCoV}$ outbreak: Similarities in the early epidemics and prediction of future trends. Chinese Medical Journal. 2020.

10. Mandal S, Bhatnagar T, Arinaminpathy N, Agarwal A, Chowdhury A, Murhekar M, Sarkar S. Prudent public health intervention strategies to control the coronavirus disease 2019 transmission in India: A mathematical model-based approach. The Indian journal of medical research. 2020;151(2-3):190. 